\title{
Sonification of flow instabilities in CFD aneurysm models
}

\author{
Daniel E. MacDonald ${ }^{1}$, Thangam Natarajan ${ }^{1}$, Richard C. Windeyer ${ }^{2}$, Peter W. Coppin ${ }^{2}$, David A. Steinman ${ }^{1}$ \\ ${ }^{1}$ Biomedical Simulation Laboratory \\ University of Toronto \\ Toronto, Canada \\ ${ }^{2}$ Perceptual Artifacts Laboratory \\ Ontario College of Art Design University \\ Toronto, Canada
}

\begin{abstract}
Recent investigations using image-based computational flow dynamics (CFD) have revealed turbulent-like blood flow instabilities (hemodynamics) within intracranial cerebral aneurysms and surrounding vasculature, which may contribute to aneurysm initiation, growth, and rupture. We describe a method derived from spectral decomposition of flow for inspecting and characterizing these "turbulent-like" hemodynamic structures in intracranial aneurysms by sonification of CFD generated data. Motivated by auscultation techniques, the current research seeks to address the limitations of conventional CFD animations by allowing the user to listen to complex flow patterns that are often difficult to discern visually. In the process, the proposed method of sonification is successfully applied to a series of datasets resulting from highfidelity numerical simulations of intracranial aneurysms. The resulting framework is shown to be highly efficient in performing parametric sonification in real time.
\end{abstract}

Keywords- CFD; biomedical; sonification; signal processing

\section{INTRODUCTION}

A cerebral aneurysm is the localized bulging of a weakened artery wall within the brain. Rupture occurs when the stresses exerted on the artery wall exceed the strength of the wall.

Blood flow instabilities (hemodynamics) are thought to contribute to the initiation, growth, and rupture of intracranial aneurysms [1], by stimulating degenerative remodeling (weakening) of the artery wall. The forces associated with these unstable flows are difficult to measure non-invasively using current technologies, thus, medical image-based CFD has been used to investigate these hemodynamic forces retroactively [1][2][3].

The initiation, growth, and rupture of these aneurysms is a complex, multifactorial process, but it is known that remodeling occurs according to flow conditions [4]. At Reynolds number of less than 500, turbulent-like flow has been observed in glass models of human intracranial aneurysms [5], starkly contrasting the commonly accepted threshold value of $\mathrm{Re}=2300$.

Using digitized patient-specific vasculature models extract-

This research was funded by the Canadian Natural Sciences and Engineering Research Council, the Social Sciences and Humanities Research Council, and the Heart \& Stroke Foundation. ed from clinical 3D angiography images, blood flow is simulated using advanced numerical methods. The results of such simulations are typically visualized as part of the process of data analysis.

These investigations have revealed turbulent-like flow instabilities within aneurysms or the vessels that host them, with implications for aneurysm rupture and initiation [1][2]. Such instabilities are typically represented through conventional visualization paradigms (glyphs, streamlines, isosurfaces), and though these can provide tangible and intuitive representations of the hemodynamics, visual differences among unstable laminar, transitional, and turbulent flow regimes in complex vascular geometries are often difficult to discern.

Auscultation refers to the established medical technique of listening to the sounds of the body for evaluation purposes [6]. For example, clinicians are trained to identify turbulent blood flows by recognizing auditory properties of duration, quality, and timing during examination of bruits in the carotid artery [6]. Doppler auscultation has been used in preliminary stages to listen to valvular movements and blood flow velocity sounds during cardiac examination, resolving sounds undetected by stethoscope [7], though to our knowledge, this technique has not been demonstrated on intracranial aneurysms.

Intracranial saccular aneurysms have been observed to emit acoustic signals (bruits) as a result of fluctuating hemodynamic flows thought to be characterized as turbulent-like, if not necessarily turbulent in the strict fluid mechanical sense [8][9][10]. These vibrations are difficult to measure in vivo due to difficulty differentiating these bruits from other bodily sounds, and as such are not a reliable source of diagnostic information [9].

Visually communicating spatiotemporal flow instabilities ignores the existing strengths of the human auditory system for resolving frequencies as compared to visual perception of spatial frequencies. For example, attempting to visually distinguish a difference of pitch between vibrating strings ignores the auditory ability to distinguish subtle pitch differences, of which [11] indicates can be as small as $0.2 \%$.

This lack of visual acuity is not only a problem of aneurysm visualization, but flow visualization in general. High frequency fluctuations require video or animation with an appropriately high frame rate (at least twice the fluctuation rate, in agreement with Nyquist's theorem) or greatly slowed video (thereby in- 
creasing time required to analyze data). This limitation becomes increasingly apparent as frequency of fluctuation increases.

Sonification is "the data-dependent generation of sound, if the transformation is systematic, objective and reproducible" [12]. The most common method of sonification, parameter mapping, involves careful mapping of data dimensions to auditory dimensions [13].

Sonification in the current work begins by decomposing the time domain values (velocity) in the frequency domain in order to discriminate regions of unstable flow. The current method allows inspection of individual frequency bands independently. Although point vector values remain the basis for our current work, the frequency domain processing between these vector values and the audio output distinguishes the current research from previous works. By sonifying the spectrogram, the envelope shape (percussive or smooth nature) is communicated along with spectral content.

In this context, a novel method originally inspired by Doppler ultrasound auscultation is presented for communicating the nature of the flow by augmenting conventional flow visualizations with data-driven sound (sonification) for the purposes of more easily identifying hemodynamic flow quality.

\section{METHOD}

High-fidelity CFD data sets produced using cases from the open-source Aneurisk database [14] serve as the basis of this methodology. These simulations were performed with a highresolution strategy where a high-fidelity Navier-Stokes solver was used in combination with fine spatial and temporal resolutions. Fully developed Womersley velocity profiles were applied at the inlet, and zero pressure was specified at the outlets.

The density of the fluid was $1.025 \mathrm{~g} / \mathrm{cm} 3$ and its corresponding viscosity 0.035 Poise was used. The computational mesh consisting of tetrahedral elements is generated using the open-source Vascular Modelling Toolkit (VMTK) [15] from segmented and preprocessed CT angiograms. A second-orderaccurate, minimally dissipative, and energy-preserving opensource CFD solver (Oasis) is used. Number of elements range from hundreds of thousands to millions of elements, with a time-step resolution on the order of $0.1 \mathrm{~ms}$ [16][17].

Within an interactive visualization environment (ParaView - an opensource scientific visualization tool), the average power spectral density of velocity-time traces is computed for a probed sample volume, defined by centre point and radius, in order to evaluate a local region of flow within the aneurysm sac. The probe radius is chosen based on two considerations: first, the real-time computation must support interactivity, and second, the probe should not be too spatially localized or too global. To define this balance of local and global volume, the upper bound of probe radius should neck diameter of the aneurysm, while the lower bound will be considered small enough to separate coherent Q-criterion structures. The frequency content and time-varying power of the resulting spectrogram are sent to a real-time audio server (SuperCollider sound server) to control the frequency and loudness of a bank of audio-rate sound generators. The summation of these generators results in a harmonically rich signal representative of the probed location.

This on-the-fly implementation is contained (though not constrained) within the ParaView Python shell and intended to run alongside a synchronized animation. Sound networking protocol Open Sound Control (OSC) is used to communicate the spectrogram data between the live ParaView environment and audio server. The audio presentation of the signal is highly configurable while maintaining audio-rate synthesis, allowing the user to pitch, slow down, or threshold by frequency band in real-time, synchronized to the visualization. Fig. 1 provides an overview of this process.

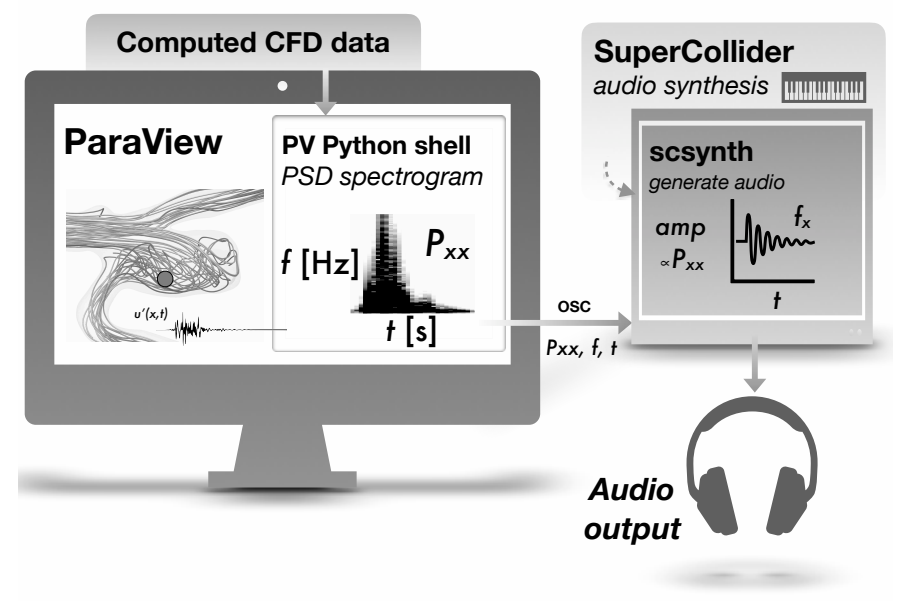

Figure 1: An overview of the method of implementation.

The structure of the sonification "instrument" was designed such that any aneurysm dataset (or any collection of spatiotemporal data) may be used as input with little manipulation (only requiring a transpose of velocity vectors). As the user probes the data, varying audio outputs are generated local to the probe. As such, different frequency content and amplitude envelopes are readily generated for each new sample of the dataset.

A guiding philosophy of sonic aesthetics was to caricature the existing physiological phenomena by generating sound inspired by fluid sounds existing in nature, and not necessarily to recreate the full acoustic field tied to the flow.

The basis of our parameter mapping was generated as simply as possible: the spectral content of velocity is mapped with auditory frequency values, while magnitude of spectral content is mapped with loudness. Use of spatial audio was considered to represent "space" of the mesh, but was ultimately found more useful for communicating the kinetic directionality of flow within the sample volume.

\section{A. Implementation}

Data is stored in binary data frames and accessed interactively from the Paraview render view. SuperCollider is used to generate the sonification from the flow data. The real-time selection, filtering, and spectral processing of flow velocity is described below. 
The chosen probe radius used is $0.25 \mathrm{~mm}$, containing approximately 50 discrete points within the volume. A list of point identifiers is generated for a 20 uniformly distributed random points within this local volume. The velocity values for these points through time will serve as the basis for the sonification, to be discussed in subsequent sections. This sonification is intended to accompany a synchronized visualization with time duration chosen by the user. As such, this pipeline is updated once for each period of flow. The data has temporal resolution of $0.004 \mathrm{~s}$ (2500 samples/second) and about 500000 points.

As shown in Fig 2, the velocity content for each point is extracted and filtered by criteria of spectral power index (SPI) to isolate flow instabilities.

SPI is a frequency domain decomposition method introduced by Khan et al as a means to quantifying the transient instabilities of turbulent-like pulsatile flows without cycle-tocycle variations [18]. This decomposition separates the pulsatile carrier signal from the turbulent-like flow instabilities. By using a high pass filter to remove the $99 \%$ most energycarrying frequencies (corresponding to a cutoff frequency of 25 $\mathrm{Hz}$ ), flow instabilities can be isolated. SPI is defined as the ratio of energy contained within the flow instabilities as a fraction of all signal energy.

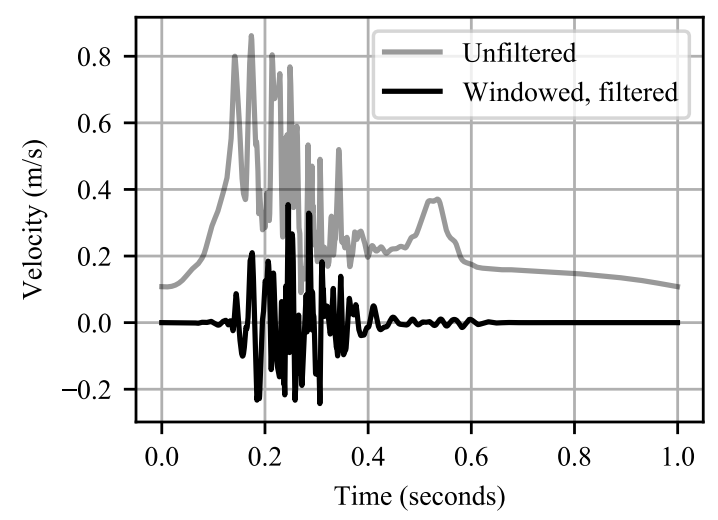

Figure 2. Isolation of velocity fluctuations by high-pass filtering the pulsatile signal according to SPI criteria. A Blackman window is used prior to filtering.

The local Power Spectral Density (PSD) spectrogram for the filtered velocity data associated with each point is calculated using a window length of 256 samples with 192 sample overlap, resulting in a smooth, temporally shifting view of the power content for each frequency bin. The average spectrogram for the sample volume is then computed. This matrix of power content corresponds to 128 frequency bins x 36 time bins.

A synthesizer template used for creating synth nodes (a synthdef) was generated in the SuperCollider environment and stored on scsynth (a real-time SuperCollider audio server). Using sound networking protocol Open Sound Control (OSC), the power matrix is sent to scsynth.

The frequency content and time-varying power of the computed spectrogram are fed to a bank of sound generators. Each frequency bin of the spectrogram corresponds to the frequency of a generator. The time-varying power values of the spectrogram are used to modulate the loudness (amplitude) of each generator in time.

The power associated with each bin corresponds to the temporal loudness (amplitude envelope) of the sound as well as a "complexity" parameter, while the frequency value corresponds to the resonant frequency of a constant skirt gain filter (Ringz generator) with noise input. The "complexity" parameter controls the cutoff frequency and modulation depth of a resonant high-pass filter. By pseudo-randomly shifting the cutoff frequency of the high pass filter near the resonant frequency of the Ringz generator, and with a shift depth proportional to the temporal power, sound textures resembling bubbles are generated to indicate a higher level of flow complexity.

The apparent directionality of flow is communicated by rotating an ambisonic B-format sound field around the user. Given the position of the aneurysm model onscreen, the directionality of flow relative to the user is determined and used to spatialize the sound; for flow visually moving left-to-right, the sound rotates correspondingly, and vice versa. The perceived rotation angle indicates the relative velocity orthogonal to the user.

This on-the-fly generation is intended to accompany a conventional flow visualization in ParaView. All computed data (signal filtering and extraction of PSD) is computed within the Paraview python shell.

\section{RESUlTS AND DISCUSSION}

A current example of the interactive implemented method can be found at [19].

The present framework of sonification results in a highly configurable audio representation allowing on-the-fly exploration of flow instabilities within CFD data. Frequency bands can be individually emphasized or extracted for closer inspection. Different sound textures, ranging from Doppler-inspired sounds to bubbly flow sounds, exist within the instrument framework. This sonification runs in real time next to a simplified conventional visualization on consumer grade hardware, allowing for flexible inspection of data.

Fig 3. illustrates a variety of flow conditions existing within an aneurysm. Velocity of flow is high entering the neck of the aneurysm, but contains minimal fluctuation. Entering the sac, it is observed that fluctuations increase substantially. The intensity of the spectral content is presented on a logarithmic color scheme and normalized for presentation. High-frequency fluctuations present within the aneurysm sac indicate disturbed flow, which may have implications for aneurysm initiation, growth, and rupture as described previously. Contours of Qcriterion for a time instant during the systole are shown for each point on a constant $y-z$ plane, where discrete locations of intense Q-criterion are observed.

The prototype has been tested with five aneurysm datasets, each of similar temporal and spatial resolution but varying flow complexity. Further exposure to new datasets will confirm the 


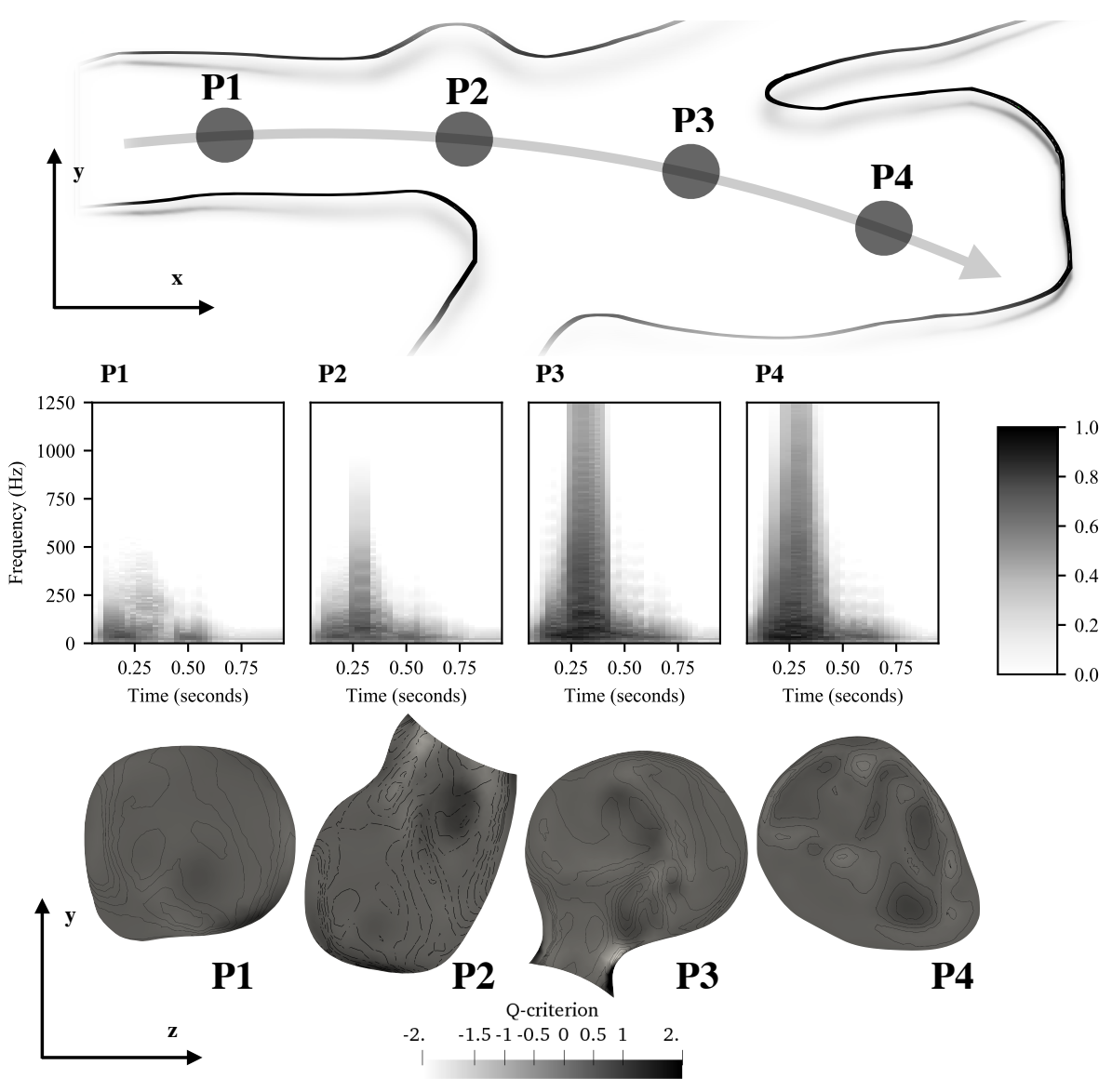

Figure 3. A comparison of spectral content and intensity of Q-criterion through the aneurysm neck and into the sac

existing method of psychological scaling, or suggest further refinement to accommodate a broader range of flow conditions.

The biological and clinical implications of high frequency content from a decomposed velocity signal are not in the scope of the current investigation; these effects are discussed in greater depth in CFD literature. The physiological presence of high frequency fluctuations, along with frequency-specific amplitude envelopes, are to be communicated to the user for interpretation. Other simplified strategies such as directly communicating Q-criterion to indicate turbulent flow were sonified, but in omitting so many dimensions of the underlying data, this simple feedback system was found too simplistic for anything other than locating regions of instability. The strength of this sonification lies in maintaining the nuance of the original signal.

The frequency resolution of the calculated PSD is limited by the temporal resolution of the data. By windowing the data, variation in time is gained at the expense of frequency resolution. This is a reasonable balance if the signals often approximate noise; broad temporal and spectral characteristics are communicated. From this balance, there are two potential dis-

advantages. First, the hard-set designation of frequency bands results in similar timbral properties between datasets. Secondly, and perhaps more importantly, if the signals have strong harmonic content, greater frequency resolution may be required to explore spectral relationships. Given the existing knowledge of spectral content in turbulent-like flow, the current chosen windowing parameters are acceptable.

The proposed method for interpreting cerebrovascular flows is not limited by application, and may be useful for other time-series spatial datasets containing instabilities. For example, we implemented a similar approach in the $3 \mathrm{D}$ creation suite Blender, for visualizing and sonifying flow around a vertical wind turbine, placing third in WestGrid's Visualize This! 2017 Challenge [20].

\section{CONCLUSION}

A novel method for sonifying CFD data for the purpose of better communicating high frequency fluctuations within simulated intracranial aneurysmal flows is described. This frequency-domain method is intended to augment conventional visualization in order to ease the interpretation of dense spatiotemporal datasets by allowing the user to visually concentrate on one field while listening to another. This method for data 
inspection builds on established analysis of intracranial hemodynamics with a focus on real-time inspection inspired by the existing field of auscultation.

\section{REFERENCES}

[1] Valen-Sendstad, Kristian, Kent-André Mardal, and David A. Steinman. 2013. "High-Resolution CFD Detects High-Frequency Velocity Fluctuations in Bifurcation, but Not Sidewall, Aneurysms." Journal of Biomechanics 46 (2):402-7.

[2] Valen-Sendstad, Kristian, Marina Piccinelli, and David A. Steinman. 2014. "High-Resolution Computational Fluid Dynamics Detects Flow Instabilities in the Carotid Siphon: Implications for Aneurysm Initiation and Rupture?" Journal of Biomechanics 47 (12):3210-16.

[3] Varble, Nicole, Jianping Xiang, Ning Lin, Elad Levy, and Hui Meng. 2016. "Flow Instability Detected by High-Resolution Computational Fluid Dynamics in Fifty-Six Middle Cerebral Artery Aneurysms." Journal of Biomechanical Engineering 138 (6):061009.

[4] Chien, Shu. 2007. "Mechanotransduction and Endothelial Cell Homeostasis: The Wisdom of the Cell." American Journal of Physiology-Heart and Circulatory Physiology 292 (3):H1209-24.

[5] Roach, M. R., S. Scott, and G. G. Ferguson. 1972. "The Hemodynamic Importance of the Geometry of Bifurcations in the Circle of Willis (glass Model Studies)." Stroke; a Journal of Cerebral Circulation 3 (3):255-67.

[6] Kusko, Meghan C., and Kathryn Maselli. 2015. "Introduction to Cardiac Auscultation." In Learning Cardiac Auscultation, 3-14.

[7] Mc Loughlin, Mario J., and Santiago Mc Loughlin. 2013. "Cardiac Auscultation: Preliminary Findings of a Pilot Study Using Continuous Wave Doppler and Comparison with Classic Auscultation." International Journal of Cardiology 167 (2):590-91.

[8] Sekhar, L. N., M. Sun, D. Bonaddio, and R. J. Sclabassi. 1990. "Acoustic Recordings from Experimental Saccular Aneurysms in Dogs." Stroke; a Journal of Cerebral Circulation 21 (8):1215-21.
[9] Ferguson, Gary G. 1970. "Turbulence in Human Intracranial Saccular Aneurysms." Journal of Neurosurgery 33 (5):485-97.

[10] Kurokawa, Y., S. Abiko, and K. Watanabe. 1994. "Noninvasive Detection of Intracranial Vascular Lesions by Recording Blood Flow Sounds." Stroke; a Journal of Cerebral Circulation 25 (2):397-402.

[11] Moore, B. C. 1973. "Frequency Difference Limens for Short-Duration Tones." The Journal of the Acoustical Society of America 54 (3):610 19.

[12] Hermann, Thomas, Andy Hunt, and John G. Neuhoff. 2011. The Sonification Handbook. Logos Verlag Berlin.

[13] Dubus, Gaël, and Roberto Bresin. 2013. "A Systematic Review of Mapping Strategies for the Sonification of Physical Quantities." PloS One 8 (12):e82491.

[14] AneuriskWeb project website, http://ecm2.mathcs.emory.edu/aneuriskweb. Emory University, Department of Math\&CS, 2012.

[15] Manini, S. and L. Antiga. Vmtk project website, http://www.vmtk.org.

[16] Khan, M. O., K. Valen-Sendstad, and D. A. Steinman. 2015. "Narrowing the Expertise Gap for Predicting Intracranial Aneurysm Hemodynamics: Impact of Solver Numerics versus Mesh and Time-Step Resolution.” AJNR. American Journal of Neuroradiology 36 (7):1310-16.

[17] Mortensen, Mikael, and Kristian Valen-Sendstad. 2015. "Oasis: A HighLevel/high-Performance Open Source Navier-Stokes Solver." Computer Physics Communications 188:177-88.

[18] Khan, Muhammad Owais, Christophe Chnafa, Diego Gallo, Filippo Molinari, Umberto Morbiducci, David A. Steinman, and Kristian ValenSendstad. 2017. "On the Quantification and Visualization of Transient Periodic Instabilities in Pulsatile Flows." Journal of Biomechanics 52 (February):179-82.

[19] MacDonald, D. Sonification of flow instabilities in aneurysm models demonstration video. 2018 https://www.youtube.com/watch?v=UmDvnPjnpV4

[20] WestGrid 2017 'Visualize This! 2017' Challenge. https://vimeo.com/246882338 